\title{
Role of Two Sets of RND-Type Multidrug Efflux Pump Transporter Genes, mexAB-oprM and mexEF-oprN, in Virulence of Pseudomonas syringae pv. tabaci 6605
}

\author{
Yuki Ichinose ${ }^{1,2 *}$, Takafumi Nishimura ${ }^{1}$, Minori Harada ${ }^{2}$, Ryota Kashiwagi ${ }^{1}$, Mikihiro Yamamoto ${ }^{1,2}$, \\ Yoshiteru Noutoshi ${ }^{1,2}$, Kazuhiro Toyoda ${ }^{1,2}$, Fumiko Taguchi ${ }^{1 \pi}$, Daigo Takemoto ${ }^{3}$, and Hidenori Matsui ${ }^{1,2}$ \\ ${ }^{I}$ Graduate School of Environmental and Life Science, Okayama University, Okayama 700-8530, Japan \\ ${ }^{2}$ Faculty of Agriculture, Okayama University, Okayama 700-8530, Japan \\ ${ }^{3}$ Graduate School of Bioagricultural Sciences, Nagoya University, Nagoya 464-8601, Japan \\ "Current address: Graduate School of Engineering, Nagoya University, Nagoya 464-8603, Japan
}

(Received on November 14, 2019; Revised on February 9, 2020; Accepted on March 6, 2020)

Pseudomonas syringae pv. tabaci 6605 has two multidrug resistance (MDR) efflux pump transporters, MexAB-OprM and MexEF-OprN. To understand the role of these MDR efflux pumps in virulence, we generated deletion mutants, $\Delta m e x B, \Delta m e x F$, and $\Delta m e x B \Delta m e x F$, and investigated their sensitivity to plant-derived antimicrobial compounds, antibiotics, and virulence. Growth inhibition assays with $\mathrm{KB}$ soft agar plate showed that growth of the wild-type (WT) was inhibited by $5 \mu \mathrm{l}$ of $1 \mathrm{M}$ catechol and $1 \mathrm{M}$ coumarin but not by other plant-derived potential antimicrobial compounds tested including phytoalexins. The sensitivity to these compounds tended to increase in $\triangle m e x B$ and $\triangle m e x B \Delta m e x F$ mutants. The $\triangle m e x B \Delta m e x F$ mutant was also sensitive to $2 M$ acetovanillone. The $\operatorname{mex} A B$ $o p r M$ was constitutively expressed, and activated in the $\Delta m e x F$ and $\Delta m e x B \Delta m e x F$ mutant strains. The swarming and swimming motilities were impaired in $\triangle m e x F$ and $\triangle m e x B \Delta m e x F$ mutants. The flood inoculation test indicated that bacterial populations in all mutant strains were significantly lower than that of WT, although all mutants and WT caused similar disease symptoms. These results indicate that MexAB-OprM

\footnotetext{
*Corresponding author.

Phone/FAX) +81-86-251-8308

E-mail)yuki@okayama-u.ac.jp

(c) This is an Open Access article distributed under the terms of the Creative Commons Attribution Non-Commercial License (http:// creativecommons.org/licenses/by-nc/4.0) which permits unrestricted noncommercial use, distribution, and reproduction in any medium, provided the original work is properly cited.
}

Articles can be freely viewed online at www.ppjonline.org. extrudes plant-derived catechol, acetovanillone, or coumarin, and contributes to bacterial virulence. Furthermore, MexAB-OprM and MexEF-OprN complemented each other's functions to some extent.

Keywords : acetovanillone, catechol, coumarin, MexABOprM, virulence

Handling Editor : Young-Su Seo

Plants respond to infection by microbial pathogens, and express defense responses, including preexisting antimicrobial compounds, phytoanticipins, and de novo production of antimicrobial compounds, phytoalexins (Morrissey and Osbourn, 1999; VanEtten et al., 2001). In order to establish infection, phytopathogens need to eliminate the effects of these antimicrobial compounds to convert the antimicrobials to ineffective structures by enzymes, to convert the structure of target proteins to those not affected by antimicrobial substances, and to extrude antimicrobials outside the cell. To extrude antimicrobials, bacteria have five structural groups of multidrug resistance (MDR) efflux pump transporters: resistance-nodulation-cell division (RND), small multidrug resistance, multi-antimicrobial extrusion, the major facilitator superfamily, and ATP-binding cassette superfamilies (Hernando-Amado et al., 2016; Li et al., 2015).

Some of the most relevant roles of RND efflux pumps so far identified include bacterial virulence, plant-bacteria interactions, transport of quorum sensing molecules, and extrusion of various kinds of toxic compounds (Alcalde- 
Rico et al., 2016; Alvarez-Ortega et al., 2013). The RND efflux pump is a tripartite complex composed of an inner membrane protein (IMP), an outer membrane protein (OMP), and a periplasmic membrane-fusion protein (MFP). The genes encoding each subunit are usually found in a single operon. In Pseudomonas syringae, there are different operons for the RND efflux pump transporter, mex $A B$ oprM and mexEF-opr $N$. The mexA and mexE encode MFP, whereas mexB and mexF encode IMP, and oprM and $\operatorname{opr} N$ encode OMP. We previously investigated the gene expression profiles in P. syringae pv. tabaci 6605 (Pta 6605) wild-type (WT) and its mutant strains by microarray analysis (Taguchi et al., 2015), and found that mexAB-oprM is constitutively expressed, whereas mexEF$o p r N$ is hardly expressed in the WT strain. However, these expressions were sometimes dramatically changed in the mutant strains. A flagellin-defective mutant $\Delta f l i C$, a flagellin-glycosylation-defective mutant $\Delta f g t 1$, and an $N$ acyl-homoserine lactone (AHL) synthase-defective mutant $\triangle p s y I$ were incapable of synthesizing AHL and upregulating mexEF-oprN expression (Sawada et al., 2018; Taguchi et al., 2015). On the other hand, $\Delta v f r$, a mutant of the virulence factor regulator, and $\triangle p i l A$, a mutant of pilin protein in type 4 pili upregulated the expression of mexAB-oprM (Taguchi and Ichinose, 2011, 2013). These results provided an insight: flagella-mediated and pili-mediated motilitydefective mutants have to adapt to a given condition and change their gene expression profile, including mex $A B$ oprM and mexEF-oprN, for survival (Sawada et al., 2018; Taguchi and Ichinose, 2011; Taguchi et al., 2015). Thus, the expressions of MDR efflux pump-related genes are responsive to environmental and genetic changes.

The mutant strains of $\triangle$ mexAB-oprM of $P$. syringae pv. phaseolicola $1448 \mathrm{~A}$, pv. syringae $\mathrm{B} 728 \mathrm{a}$, and pv. tomato DC3000 had reduced populations after inoculation into their own host leaves, indicating that MexAB-OprM contributes to virulence (Stoitsova et al., 2008). The susceptibilities of these mutants to aminoglycosides, $\beta$-lactams, chloramphenicol, ethidium bromide, fluoroquinolones, macrolides, nitrofurantoin, quinolones, rifampin, and tetracycline were increased, indicating that these compounds are potential substrates of MexAB-OprM (Fernando and Kumar, 2013; Stoitsova et al., 2008). Characterization of an RND transporter system in $P$. syringae pv. syringae B301D, the PseABC efflux system, revealed that PseABC has an important role in secretion of syringomycin and syringopeptin, phytotoxins of this pathogen (Kang and Gross, 2005). However, the role of RND efflux pumps in the secretion of the plant antimicrobial metabolites is not clear yet. In this study, we generated two single mutants for two major RND efflux pumps, $\triangle \operatorname{mex} B$ and $\triangle m e x F$, and a double mutant, $\triangle \operatorname{mex} B \Delta \operatorname{mex} F$, and investigated the growth inhibition effect of plant-derived antimicrobials, such as phytoanticipins and phytoalexins. Furthermore, the effects

Table 1. Bacterial strains and plasmids used in this study

\begin{tabular}{|c|c|c|}
\hline Bacterial strain or plasmid & Relevant characteristics & Reference or source \\
\hline \multicolumn{3}{|l|}{ Escherichia coli strain } \\
\hline $\mathrm{DH} 5 \alpha$ & $\begin{array}{l}F^{-}, \lambda^{-}, ø 80 d L a c Z \Delta M 15, \Delta(\text { lacZYA-argF }) \mathrm{U} 169, \text { recA1, endA1, } \\
h s d R 17\left(\mathrm{r}_{\mathrm{K}}^{-} \mathrm{m}_{\mathrm{K}}^{+}\right), \text {supE44, thi-1, gyrA, relA1 }\end{array}$ & Takara Bio \\
\hline $\mathrm{S} 17-1$ & Thi, pro, hsdR,$h s d M^{+}$, recA [chr::RP4-2-Tc::Mu-Km::Tn7] & Schäfer et al. (1994) \\
\hline $\begin{array}{l}\text { Chromobacterium violaceum } \\
\text { CV026 }\end{array}$ & $\begin{array}{l}\text { Double mini-Tn5 mutant from C. violaceum ATCC 31532; AHL } \\
\text { biosensor, } \mathrm{Km}^{\mathrm{r}}\end{array}$ & McClean et al. (1997) \\
\hline \multicolumn{3}{|l|}{ P. syringae pv. tabaci } \\
\hline Isolate 6605 & Wild-type, $\mathrm{Nal}^{\mathrm{r}}$ & Taguchi et al. (2006) \\
\hline$\Delta h r c C$ & Isolate $6605 \Delta h r c C, \mathrm{Nal}^{\mathrm{r}}$ & Marutani et al. (2005) \\
\hline$\Delta \operatorname{mexB}$ & Isolate $6605 \Delta \operatorname{mex} B, \mathrm{Nal}^{\mathrm{r}}$ & This study \\
\hline$\Delta \mathrm{mexF}$ & Isolate $6605 \Delta m e x F, \mathrm{Nal}^{\mathrm{r}}$ & Sawada et al. (2018) \\
\hline$\Delta \operatorname{mex} B \Delta \operatorname{mexF}$ & Isolate $6605 \Delta m e x B \Delta m e x F, \mathrm{Nal}^{\mathrm{r}}$ & This study \\
\hline \multicolumn{3}{|l|}{ Plasmid } \\
\hline pCR Blunt II TOPO & Cloning vector for blunt ended PCR products, $\mathrm{Km}^{\mathrm{r}}$ & Thermo Fisher Scientific \\
\hline pCR-mexAB-oprM & pCR Blunt II TOPO with 5,789-bp of mexAB-oprM, $\mathrm{Km}^{\mathrm{r}}$ & This study \\
\hline $\mathrm{pCR}-\Delta \operatorname{mex} B$ & mex $B$-deleted plasmid from pCR-mexAB-opr $M, \mathrm{Km}^{\mathrm{r}}$ & This study \\
\hline pK18mobsacB & Small mobilizable vector, $\mathrm{Km}^{\mathrm{r}}$, sucrose-sensitive $(\operatorname{sac} B)$ & Schäfer et al. (1994) \\
\hline $\mathrm{pK} 18 \Delta m e x B$ & pK18mobsacB with mexA-oprM, $\mathrm{Km}^{\mathrm{r}}$ & This study \\
\hline
\end{tabular}

AHL, $N$-acyl-homoserine lactone; $\mathrm{Km}^{\mathrm{r}}$, kanamycin resistance, $\mathrm{Nal}^{\mathrm{r}}$, nalidixic acid resistance. 
of the mutation on the expression of these RND pump genes, motility, and virulence were characterized. The roles of MexAB-OprM and MexEF-OprN in bacterial virulence are discussed.

\section{Materials and Methods}

Bacterial strains and growth conditions. All bacterial strains used in this study are listed in Table 1. Pta 6605 strains, Escherichia coli strains, and Chromobacterium violaceum CV026 were grown as described previously (Ichinose et al., 2020).

Generation of $\triangle m e x B$ and $\triangle m e x B \Delta m e x F$ mutants in Pta 6605. An approximately $5.8 \mathrm{~kb}$ DNA fragment encoding mexAB-oprM was amplified with a set of primers, mexA5 and oprM3, using KOD FX DNA polymerase (Toyobo, Osaka, Japan) with the genomic DNA of Pta 6605 as a template, then introduced into a pCR-Blunt II-TOPO plasmid vector (Invitrogen, Carlsbad, CA, USA) to obtain pCR-mexAB-oprM (Fig. 1A). To delete the mexB region from the plasmid, inverse PCR was carried out with a set of primers, mexB-d5NheI and mexB-d3NheI, and self-ligated by a Ligation-Convenience kit (Nippon Gene, Tokyo, Japan) after NheI and $D p n I$ digestions. The mutated genetic region was introduced into pK18mobsacB (Schäfer et al., $1994)$ via an $E c o$ RI site to generate pK18- $\Delta m e x B$, then introduced into E. coli S17-1 for conjugation with Pta 6605 strains. Deletion mutants were obtained by conjugation and homologous recombination according to the previously reported methods (Taguchi et al., 2006). The DNA sequence of the mutated region in the bacterium was confirmed by DNA sequencing with a Big Dye terminator cycle sequencing kit and an ABI PRISM 3100 sequencer (Thermo Fisher Scientific, Waltham, MA, USA). Primer sequences were designed based on the sequence of Pta 6605 provided by Dr. Studholme, University of Exeter, UK.

Using a previously generated $\triangle m e x F$ mutant (Sawada et al., 2018) as a recipient, $\operatorname{mex} B$ was deleted by a conjugation and homologous recombination as described above, and the $\Delta$ mex $B \Delta$ mexF double mutant was generated.

In vitro growth assay. An overnight culture was centrifuged at $1,500 \times g$ for $5 \mathrm{~min}$ at $4^{\circ} \mathrm{C}$, the bacterial pellet was resuspended in $\mathrm{LB}$ medium with $10 \mathrm{mM} \mathrm{MgCl}$, and the $\mathrm{OD}_{660}$ was adjusted to 0.02 . Three $\mathrm{ml}$ of the bacterial culture was incubated at $27^{\circ} \mathrm{C}$ with shaking, and the bacterial $\mathrm{OD}_{660}$ was measured with an OD meter at each time point.

Potential plant antibiotics. Potential antibiotics, trans-
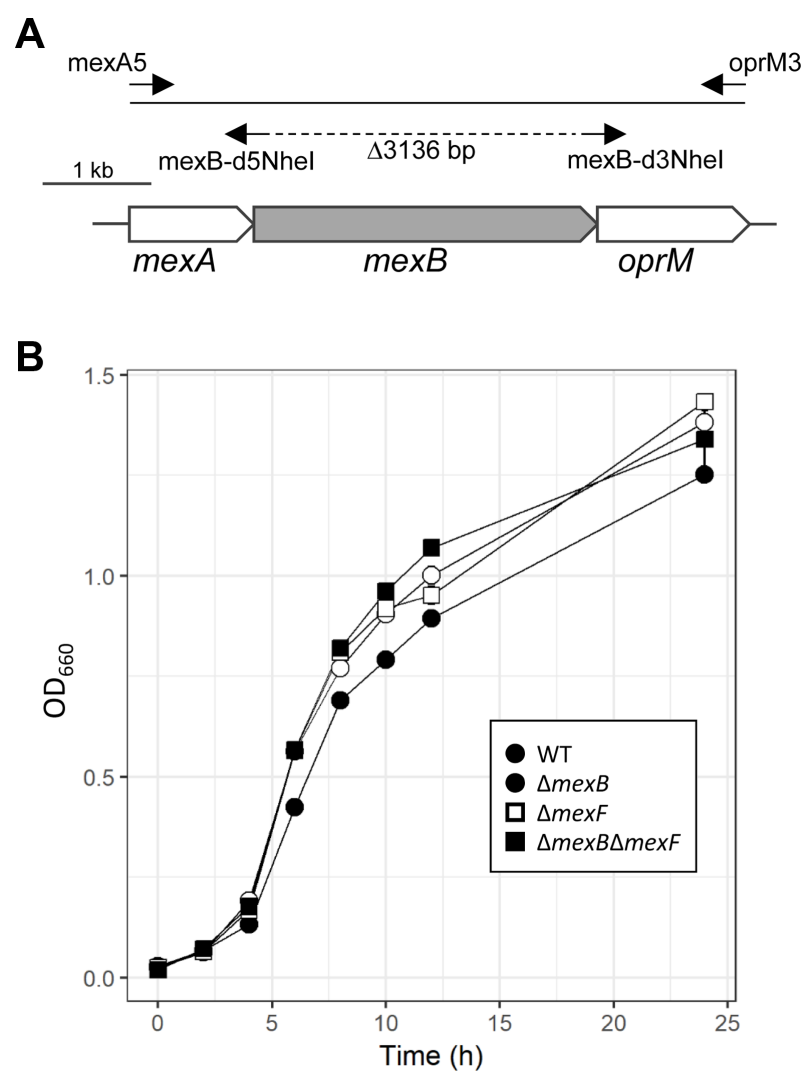

Fig. 1. Generation of $\triangle \operatorname{mex} B$ mutant stain and in vitro bacterial growth. (A) Schematic organization of mexAB-oprM in Pseudomonas syringae pv. tabaci 6605 . PCR was carried out to isolate and clone the entire region of mexAB-oprM. Using pCR-mex $A B$ oprM as a template and mexB-d5NheI and mexB-d3NheI as primers, inverse PCR was carried out to obtain pCR- $\Delta$ mexB. (B) In vitro growth of each bacterium. The same density of bacterium $\left(\mathrm{OD}_{660}=0.02\right)$ was used to incubate at $27^{\circ} \mathrm{C}$ with shaking, and bacterial $\mathrm{OD}_{660}$ was measured. The bars represent standard deviations for three independent experiments.

cinnamic acid, naringenin, acetovanillone, $(+)$-catechin, catechol, scopoletin, and coumarin were obtained from Tokyo Chemical Industry Co. Ltd. (Tokyo, Japan), phloretin, chlorogenic acid, and $p$-coumaric acid were obtained from Sigma-Aldrich (St. Louis, MO, USA), and acetosyringone was obtained from Cayman Chemical (Ann Arbor, MI, USA). Phytoalexins, capsidiol, debneyol, and rishitin were purified in the plant pathology laboratory of Nagoya University.

Growth inhibition zone. Potential antibiotics and antibiotics were dissolved in sterilized and distilled water or dimethyl sulfoxide. Bacteria $(3 \mathrm{ml})$ cultured overnight in King's B (KB) medium were mixed with $50 \mathrm{ml}$ of melted $0.6 \%$ agar KB medium, then poured into three Petri dishes. 
Table 2. Oligonucleotide sequences used in this study

\begin{tabular}{lll}
\hline Oligonucleotide name & \multicolumn{1}{c}{ Sequence $\left(5^{\prime}-3^{\prime}\right)$} & \multicolumn{1}{c}{ Purpose } \\
\hline mexA5 & TTGAGACAACAGGCTCCTGC & PCR to amplify 5,789 bp of mexAB-oprM for cloning \\
oprM3 & TCAGAACAGCTGAACCGAAGG & \\
mexB-d5NheI & ctagctagcTTACTCCCCTTTGCTGCCTG & Inverse PCR to delete mexB coding region to generate $\Delta m e x B$ \\
mexB-d3NheI & ctagctagcATGAGCAAGTCATTGATCTCT & \\
mexA-R1 & CAGGTCAACGGCATCATTCT & qRT-PCR to amplify 168 bp of $m e x A$ fragment \\
mexE-F1 & GACCAGTTGCTTGTAGCGATC & \\
mexE-R1 & CACCTGGGCCAGATGAACTT & qRT-PCR to amplify 203 bp of mexE fragment \\
pcaG-F & AGCACAAACTTCTTGCCCAG & \\
pcaG-R & TGCAGGAAACCCCTTCGC & qRT-PCR to amplify 113 bp of $p c a G$ fragment \\
\hline
\end{tabular}

Small letters indicate additive nucleotides containing artificial NheI sites in mexB-d5NheI and mexB-d3NheI.

Compound-absorbed paper discs were put on the Petri dish, and the dishes were incubated at $27^{\circ} \mathrm{C}$. After $24 \mathrm{~h}$ incubation, the growth inhibition zone was photographed and measured using Adobe Photoshop CC version 19.1.9 (Adobe, San Jose, CA, USA). The photographic data was gray-scaled, then noise was removed with a high-pass filter to a middle value of brightness, the image was converted into two gradations, and the growth inhibition zone was measured.

Extraction of total RNA and quantitative reverse transcription PCR (qRT-PCR). Overnight cultured bacteria were harvested by centrifugation and further incubated in MMMF medium (50 $\mathrm{mM}$ potassium phosphate buffer, 7.6 $\mathrm{mM}\left(\mathrm{NH}_{4}\right)_{2} \mathrm{SO}_{4}, 1.7 \mathrm{mM} \mathrm{MgCl}_{2}$, and $1.7 \mathrm{mM} \mathrm{NaCl}, \mathrm{pH} 5.7$, supplemented with $10 \mathrm{mM}$ each of mannitol and fructose) for an additional $1 \mathrm{~h}$.

Total RNA was extracted from the cells collected by centrifugation using Trizol (Thermo Fisher Scientific). For qRT-PCR, the cDNA was synthesized using $0.5 \mu \mathrm{g}$ of total RNA and ReverTra Ace qPCR RT Master Mix with gDNA Remover (Toyobo) according to the manufacturer's instructions. The specific primer sets for mexA (mexA-F1 and mexA-R1), mexE (mexE-F1 and mexE-R1), and pcaG (pcaG-F and pcaG-R) were designed according to the registered sequences of Pta 6605 (Table 2). Real-time qRTPCR was carried out using a GVP-9600 (Shimadzu, Kyoto, Japan) and KAPA SYBR FAST qPCR Master Mix (2×) Kit (Sigma-Aldrich), with primers at a final concentration of $0.4 \mu \mathrm{M}$ each and $1 \mu \mathrm{l}$ of cDNA product as a template in a $20 \mu \mathrm{l}$ reaction mixture. PCR was performed with one denaturation cycle of $20 \mathrm{~s}$ at $95^{\circ} \mathrm{C}$ and 40 cycles of $15 \mathrm{~s}$ at $95^{\circ} \mathrm{C}$ and $30 \mathrm{~s}$ at $62^{\circ} \mathrm{C}$. The expression of each gene was normalized by $p c a G$, which encodes protocatechuate 3,4-dioxygenase subunit alpha, as a stably expressed gene
(Taguchi et al., 2015). Melting curve analysis confirmed that each PCR was a single product. The PCRs were performed with a high linearity. The data were analyzed using the $\Delta \Delta C_{\mathrm{T}}$ (cycle threshold) method.

Virulence test. An inoculation test of Pta 6605 was conducted with a flood assay (Ishiga et al., 2011) optimized for tobacco plants (Nicotiana tabacum L. cv. Xanthi). Tobacco seeds were sterilized and sown on $0.8 \%$ Murashige-Skoog (MS) agar plates containing 1\% sucrose and vitamin stock solution (thiamin hydrochloride $3 \mathrm{mg} / 1$, nicotinic acid $5 \mathrm{mg} /$ 1, pyridoxine hydrochloride $0.5 \mathrm{mg} / \mathrm{l}$ ), and grown at $28^{\circ} \mathrm{C}$ under $16 \mathrm{~h}$ light: $8 \mathrm{~h}$ dark conditions for 2 weeks. Tobacco seedlings were transplanted to $0.8 \%$ MS agar plates containing $0.1 \%$ sucrose and vitamin stock solution as above and grown for 2 days under the same conditions. Bacteria were grown overnight at $27^{\circ} \mathrm{C}$ in $\mathrm{KB}$ medium with $50 \mu \mathrm{g} /$ $\mathrm{ml}$ of nalidixic acid (Nal). The bacterial inoculum was adjusted to $\mathrm{OD}_{600}=0.002$ with sterilized water containing 0.025\% Silwet L-77 (Biomedical Sciences, Tokyo, Japan). Sterilized water containing $0.025 \%$ Silwet L-77 was used as a mock treatment. The inoculation test was carried out on a clean bench. The bacterial suspension (approximately $30 \mathrm{ml}$ ) was poured into the plate of tobacco seedlings. After about $10 \mathrm{~s}$ incubation, the bacterial suspension was decanted, and the plate was air-dried on a clean bench for 15 $\mathrm{min}$. The plants were incubated under $16 \mathrm{~h}$ light: $8 \mathrm{~h}$ dark conditions at $22^{\circ} \mathrm{C}$, and disease symptoms were observed for 4 days post-inoculation (dpi) and $10 \mathrm{dpi}$. To determine the bacterial population at $0 \mathrm{dpi}$ and $4 \mathrm{dpi}$, leaf disks were punched out using a disposable biopsy hole punch and then ground with mortar and pestle. The homogenates were serially diluted in sterile distilled water and then spread on $\mathrm{KB}$ plates containing Nal. The plates were dried and incubated at $27^{\circ} \mathrm{C}$ for 2 days, after which the bacterial population 
was measured by counting the number of colonies (colony forming unit).

Swimming and swarming motilities. Swimming and swarming assays were performed as described previously (Taguchi and Ichinose, 2011). An overnight culture with 50 $\mu \mathrm{g} / \mathrm{ml}$ of Nal was centrifuged at $1,500 \times g$ for $10 \mathrm{~min}$, the bacterial pellet was resuspended in $10 \mathrm{mM} \mathrm{MgSO}_{4}$, and the $\mathrm{OD}_{600}$ was adjusted to 1.0. For the swimming assay, $3 \mu \mathrm{l}$ of bacterial suspension was inoculated on the center of $0.25 \%$ Bactoagar (Becton Dickinson \& Company, Glencoe, MD, USA) (w/v) MMMF plates. For the swarming assay, $3 \mu \mathrm{l}$ of the bacterial suspension was spotted on the center of a $0.45 \%$ Bactoagar (w/v) SWM $(0.5 \%$ peptone and $0.3 \%$ yeast extract) plate and allowed to dry. Plates were incubated overnight at $23^{\circ} \mathrm{C}$. Swimming and swarming motilities were observed after $24 \mathrm{~h}$ incubations.

Detection of $N$-acyl homoserine lactones. Bacterial strains were grown in $\mathrm{KB}$ medium for $24 \mathrm{~h}$ at $27^{\circ} \mathrm{C}$. AHLs were extracted with an equal volume of ethyl acetate and detected using $\mathrm{C}_{18}$ reversed-phase thin layer chromatography (TLC Silica gel 60, Merck, Darmstadt, Germany) and the biosensor C. violaceum CV026 (Taguchi et al., 2006) as described in Ichinose et al. (2020).

Statistical analysis. Statistical analysis was performed using One-way ANOVA Dunnett's test and Tukey honestly significant difference test with $\mathrm{R}$ software version 3.6.1 (https://www.r-project.org/).

\section{Results}

Generation of deletion mutants $\triangle \operatorname{mex} B, \Delta \operatorname{mex} F$, and $\triangle m e x B \Delta m e x F$ in Pta 6605. The specific deletion of mexB and/or mexF was confirmed by genomic PCR (data not shown). The growth of each bacterial strain in in vitro culture was compared. There was no significant difference in growth speed between the WT strain and the mutant strains (Fig. 1B).

Growth inhibition by plant-derived antimicrobial compounds and antibiotics in WT and mutant strains. Growth inhibition assays of the plant-derived potential antimicrobial compounds in KB soft agar plates showed that $5 \mu \mathrm{l}$ of $0.9 \mathrm{M}$ trans-cinnamic acid, $0.5 \mathrm{M}$ p-coumaric acid, $1 \mathrm{M}$ acetosyringone, $1 \mathrm{M}$ scopoletin, $1 \mathrm{M}$ chlorogenic acid, $0.3 \mathrm{M}$ phloretin, $1 \mathrm{M}$ naringenin, and $200 \mathrm{mg} / \mathrm{ml}(+)$-catechin did not produce any clear growth inhibition zone in all tested strains (data not shown). Furthermore, $5 \mu 1$ of 500

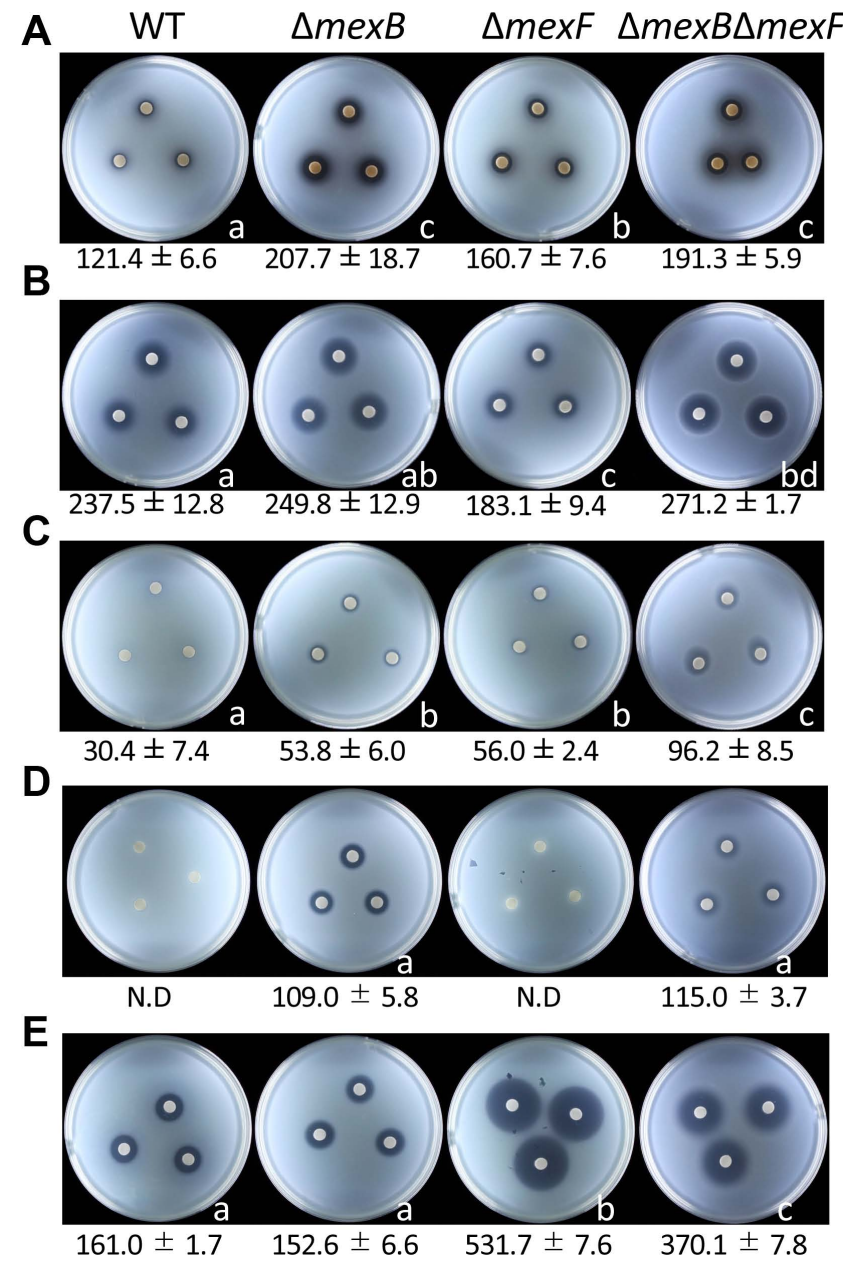

Fig. 2. Sensitivity of Pseudomonas syringae pv. tabaci 6605 wild-type (WT) and its mutant strains to several antimicrobial compounds. Growth inhibition on plates with $5 \mu \mathrm{l}$ of $1 \mathrm{M}$ catechol (A), $1 \mathrm{M}$ coumarin (B), $2 \mathrm{M}$ acetovanillone (C), $50 \mu \mathrm{g} /$ $\mathrm{ml}$ ampicillin (D), and $50 \mu \mathrm{g} / \mathrm{ml}$ chloramphenicol (E). N.D., not detected. Photographs were taken at $24 \mathrm{~h}$ after incubation. Data shown below photographs are the averages of three independent replicates of growth-inhibited areas $\left(\mathrm{mm}^{2}\right)$. Statistical groups were determined using Tukey's honestly significant difference test $(P<0.05)$, and significant differences are indicated by different letters below right of each photograph.

$\mu \mathrm{M}$ capsidiol, debneyol, and rishitin, phytoalexins of Nicotiana tabacum, Nicotiana debneyi, and Solanum tuberos$u m$, respectively, also did not inhibit any bacterial growth (Supplementary Fig. 1). When the concentration of capsidiol was increased to $10 \mathrm{mM}$, no growth inhibition was observed (Supplementary Fig. 1). This concentration of phytoalexins completely inhibits the growth of oomycetes and fungal pathogens (Egea et al., 1996). However, $5 \mu \mathrm{l}$ of $1 \mathrm{M}$ catechol and $1 \mathrm{M}$ coumarin inhibited the growth of the Pta 6605 WT strain (Fig. 2A and B). Furthermore, the size 
of the growth inhibition zone by $1 \mathrm{M}$ catechol increased in $\triangle m e x F$, and further expanded in $\triangle m e x B$ and $\triangle m e x B \Delta m e x F$. The $\triangle$ mex $B$ and $\triangle$ mex $B \Delta$ mexF mutant strains showed a slightly higher sensitivity than the WT strain to $1 \mathrm{M}$ coumarin; however, the $\Delta m e x F$ mutant produced a smaller inhibition zone than the WT strain. Although $5 \mu \mathrm{l}$ of $2 \mathrm{M}$ acetovanillone did not inhibit growth of the WT strain, it inhibited those of $\triangle m e x B$ and $\triangle m e x F$, and an additive growth inhibition effect was observed in the $\Delta$ mex $B \Delta$ mexF mutant (Fig. 2C).

The sensitivity of bacterial strains to some bacteriaderived antibiotics was also investigated. The sensitivities to most of the antibiotics used in this study, such as kanamycin, spectinomycin, tetracycline, streptomycin, and hygromycin $\mathrm{B}$, did not differ among the bacterial strains (data not shown). However, only $\triangle m e x B$ and $\Delta m e x B \Delta m e x F$ were sensitive to $5 \mu \mathrm{l}$ of $50 \mathrm{mg} / \mathrm{ml}$ ampicillin, while the growths of the WT strain and $\Delta m e x F$ were not inhibited by it (Fig. 2D). On the contrary, although the WT and $\triangle \operatorname{mex} B$ mutant strains showed only weak growth inhibition zone to $5 \mu \mathrm{l}$ of $50 \mathrm{mg} / \mathrm{ml}$ chloramphenicol, the growth of $\Delta m e x F$ and $\Delta$ mex $B \Delta$ mex $F$ mutant strains was remarkably inhibited (Fig. 2E). These results indicate that MexAB-OprM and MexEF-OprN specifically extrude ampicillin and chloramphenicol, respectively.

Gene expression profiles of mex $A$ and mexE in $\triangle m e x B$, $\Delta m e x F$, and $\triangle$ mexB $\Delta$ mex $F$ mutants. The effect of the deletion of mexB, mexF, or both mexB/mexF on mexA and mexE gene expression was analyzed by real-time qRTPCR. As shown in Fig. 3A, the expression of $\operatorname{mex} A$ was enhanced in the $\triangle m e x F$ and $\Delta m e x B \Delta m e x F$ strains. In addi-
A

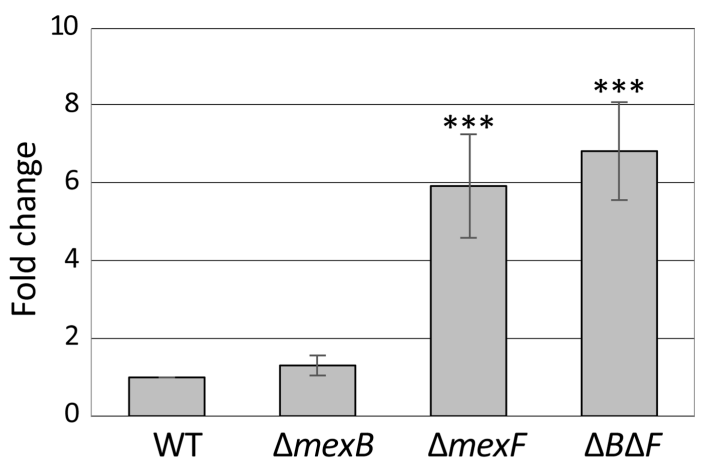

B

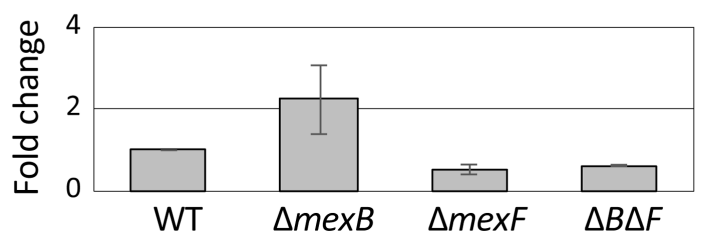

Fig. 3. Quantitative reverse transcription PCR analysis of gene expression of $\operatorname{mexA}(\mathrm{A})$ and $\operatorname{mex} E(\mathrm{~B})$ in wild-type (WT) and mutant strains. Asterisk indicates significant difference from the WT $(* * * P<0.001)$ determined by one-way ANOVA Dunnett's test.

tion, the expression of mexE was very weak, and seemed to be slightly increased in the $\Delta m e x B$ strain (Fig. 3B).

Motility and AHL production in WT and mutant strains. Surface swimming and swarming motilities of the $\triangle$ mexB mutant were comparable to WT strain, whereas those of $\triangle m e x F$ and $\Delta m e x B \Delta m e x F$ double mutant strains were abolished (Fig. 4A).
A

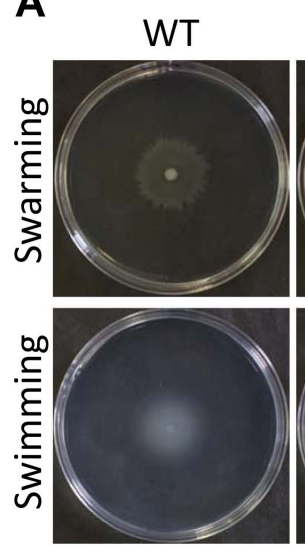

$\Delta \operatorname{mex} B$

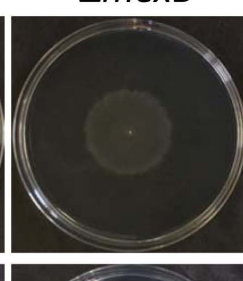

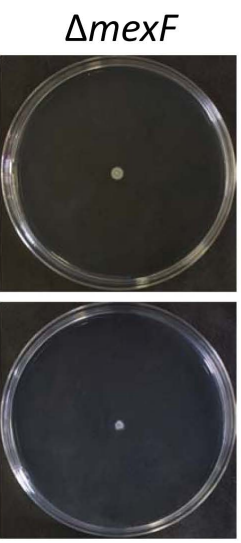

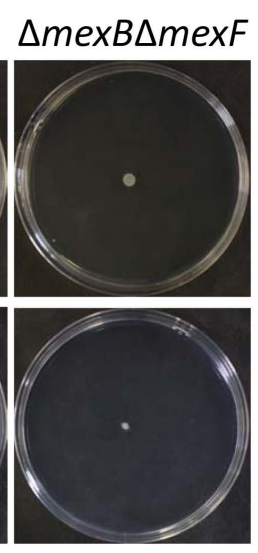

B

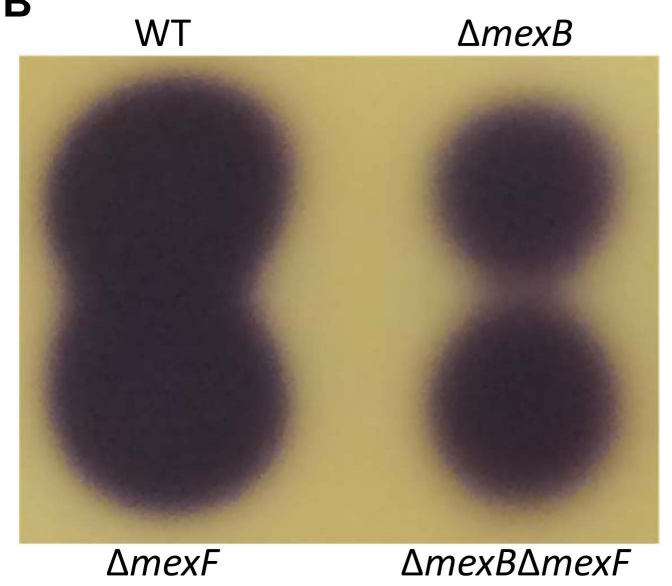

Fig. 4. Swarming and swimming motilities and $N$-acyl-homoserine lactone (AHL) production. (A) Surface swarming and swimming motilities after $24 \mathrm{~h}$ incubation. (B) AHL production. Ethyl acetate extract from $0.2 \mathrm{ml}$ of each bacterial culture was spotted on the thin layer chromatography plate. AHL was visualized as described in "Materials and Methods". 
A
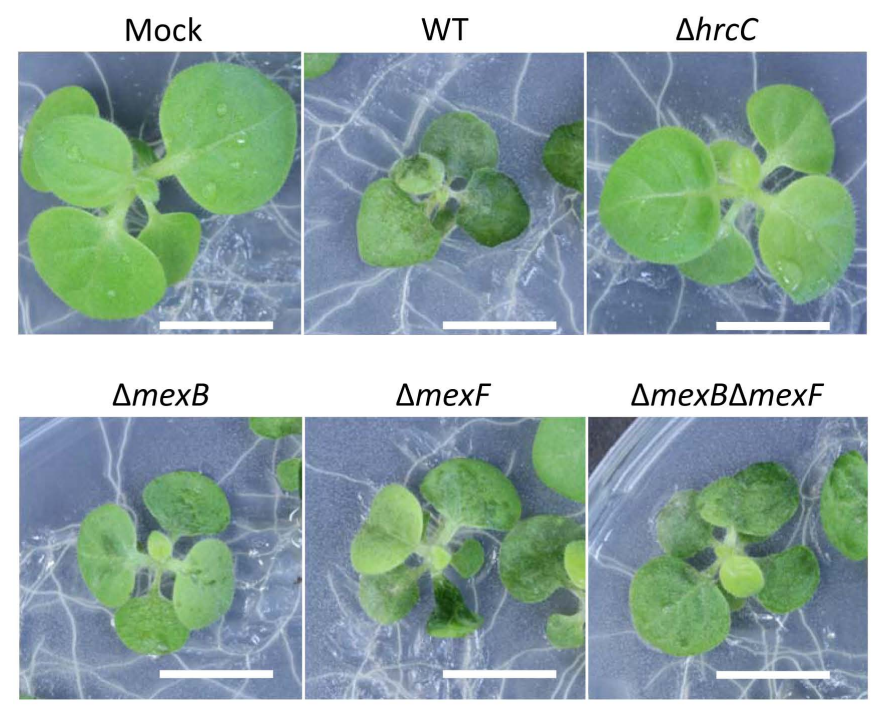

B

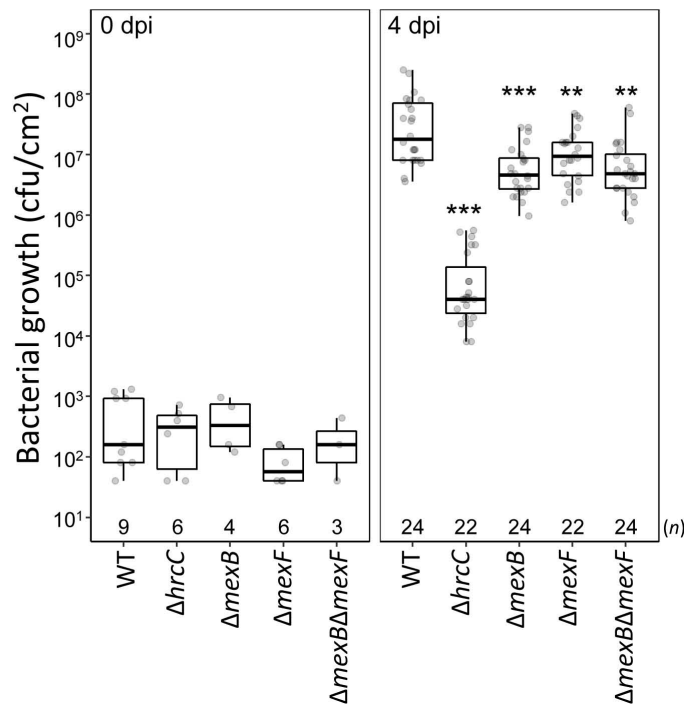

Fig. 5. Inoculation test. (A) Tobacco seedlings were inoculated with wild-type (WT) or $\Delta h r c C, \Delta \operatorname{mexB}, \Delta \operatorname{mexF}, \Delta \operatorname{mex} B \Delta \operatorname{mexF}$, and $\triangle h r c C$ strains and incubated at $23^{\circ} \mathrm{C}$. Photographs taken 4 days post-infection (dpi) shows representative results. Scale bars $=1 \mathrm{~cm}$. (B) Bacterial populations were determined at 0 or 4 dpi. Box plots and Jitter plots representing the bacterial growth in inoculated plants from three biological replicates. Boxes show the upper and lower quartiles of the data, and horizontal black lines represent the medians. Jitter plots indicate raw data. Statistical significance was determined using one-way ANOVA Dunnett's test where each group was compared with the WT. $\left({ }^{* *} P<0.01,{ }^{* * *} P<0.001\right) . n$ is shown above the stain names.

Pta 6605 utilizes AHLs as quorum-sensing molecules. AHLs production in $\triangle \operatorname{mex} B$ and $\triangle \operatorname{mex} B \Delta \operatorname{mexF}$ mutants was reduced. However, the $\triangle m e x F$ mutant retained AHL production ability as good as that of the WT strain (Fig. 4B).

Virulence of WT and mutant strains. To investigate whether MexAB-OprM and/or MexEF-OprN of Pta 6605 is involved in the virulence, a flood inoculation test of the WT, $\Delta$ mexB $, \Delta \operatorname{mexF}, \Delta \operatorname{mex} B \Delta \operatorname{mex} F$, and $\Delta h r c C$ mutant strains (Marutani et al., 2005) on host tobacco seedlings was performed. The $\Delta h r c C$ mutant did not show any disease symptoms, and bacterial growth was limited (Fig. 5). On the other hand, all mutants except $\Delta h r c C$ showed disease symptoms similar to those of the WT strain at 4 dpi (Fig. 5A) and 10 dpi (Supplementary Fig. 2). However, the bacterial populations in the leaves of $\triangle \operatorname{mex} B, \Delta \operatorname{mex} F$, and $\Delta$ mex $B \Delta$ mexF mutant strains were significantly lower than that of WT at 4 dpi (Fig. 5B), indicating that MexABOprM and MexEF-OprN might partially contribute to virulence at the early stage of infection.

\section{Discussion}

Stoitsova et al. (2008) reported that deletion mutants of
mexAB-oprM of $P$. syringae pv. tomato DC3000, pv. phaseolicola 1448A, and pv. syringae B728a exhibited increased susceptibility to bacterial and plant-derived antimicrobials, detergents, and dyes. In this study, we focused on the functions of MexAB-OprM and MexEFOprN on the plant-derived antimicrobials, phytoanticipins and phytoalexins in $P$. syringae pv. tabaci. The increased growth inhibition in $\triangle m e x B$ and $\Delta m e x B \Delta m e x F$ of Pta 6605 by acetovanillone, catechol, and coumarin indicates that MexAB-OprM may extrude these compounds. Baker et al. (2015) reported that tobacco leaves accumulated acetovanillone, acetosyringone, and chlorogenic acid in response to the inoculation of $P$. fluorescens, $P$. syringae pv. tabaci, and $P$. syringae pv. syringae. Therefore, Pta 6605 may extrude these phytoanticipins during infection. Analysis of growth inhibition by formation of growth inhibition zones has the advantage that even hydrophobic substances can be absorbed in a paper disc and their antimicrobial activity examined. However, we did not observe antimicrobial activity in many plant-derived compounds with this method. Therefore, it is possible that some of these compounds might show an inhibitory activity with other methods.

It was reported that the expression of mexAB-oprM of $P$. syringae pv. tomato DC3000 increases in the presence of flavonoids such as phloretin and naringenin (Vargas et al., 
2011). The mexA mutant exhibits higher susceptibility to these compounds and caused fewer symptoms compared to the WT strain in the inoculation test on tomato leaves (Vargas et al., 2011). Furthermore, the mexA mutant bore fewer flagella, and the presence of phloretin reduced the ratio of flagellated cells and swarming motility (Vargas et al., 2013). These results indicate that the MexAB-OprM transporter contributes to the colonization on host tomato plants. Although we observed similar swimming and swarming motilities in WT and $\triangle$ mexB mutant, the mutation of mexF resulted in the loss of these motilities, indicating relationship between MexEF-OprN and motility.

An extract of Arabidopsis thaliana contains 4-methylsulfinylbutyl isothiocyanate, a natural product derived from aliphatic glucosinolates, which inhibited the growth of nonhost Pseudomonas bacteria (Fan et al., 2011). In contrast, $P$. syringae pathovars like $P$. syringae pv. tomato DC3000 that are pathogenic to $A$. thaliana can grow with the same extract from Arabidopsis because these pathovars have the sax (survival in Arabidopsis extract) genes. Parts of the sax genes encode MexAB-OprM and MexEF-OprN and confer the ability to extrude a wide range of substrates including antibiotics and host-derived molecules (Fan et al., 2011). The major phytoanticipin varies from plant to plant. Because acetovanillone, catechol, and coumarin inhibit the growth of Pta 6605, these compounds may be major phytoanticipins of tobacco, and both MexAB-OprM and MexEF-OprN contribute to the virulence of Pta 6605.

As Gnanamanickam and Mansfield (1981) already reported that isoflavonoid (kievitone and phaseollin), flavonoid (hydroxyflavans), furanoacetylenic (wyerone), and sesquiterpenoid (capsidiol and rishitin) phytoalexins do not inhibit the growth of a wide range of bacteria, none of the phytoalexins tested also showed an inhibitory effect on Pta 6605 or even $\triangle \operatorname{mex} B, \Delta \operatorname{mex} F$, and the $\Delta$ mexB $\Delta \operatorname{mex} F$ double mutant (Supplementary Fig. 1). Fifty $\mu \mathrm{g}$ of capsidiol and rishitin did not inhibit the growth of six Gram-positive and eight Gram-negative bacteria including saprophytes and plant and animal pathogens. Camalexin, a phytoalexin in $A$. thaliana, was accumulated in response to the inoculation of $P$. syringae pv. syringae (Tsuji et al., 1992). However, a defective mutant that cannot synthesize the camalexin of $A$. thaliana still exhibits resistance to avirulent $P$. syringae pathogens, indicating that the accumulation of camalexin is not major defense response to bacterial pathogens (Glazebrook and Ausubel, 1994). These mutants displayed enhanced sensitivity to virulent $P$. syringae strains, suggesting that camalexin limits the growth of virulent bacteria (Glazebrook and Ausubel, 1994).

The mexEF-oprN of the WT strain is hardly expressed in the general culture conditions. However, it was weakly activated in the $\triangle \operatorname{mex} B$ mutant. MexEF-OprN might compensate for the lost functionality of MexAB-OprM. MexAB-OprM and MexEF-OprN were expected to extrude acetovanillone, catechol, and coumarin. We will now investigate whether these compounds induce expressions of mexAB-oprM and mexEF-opr $N$ genes.

The virulence of $\triangle m e x B, \Delta m e x F$, and $\triangle m e x B \Delta m e x F$ was reduced (Fig. 5), and swimming and swarming motilities of $\triangle m e x F$ and $\triangle m e x B \Delta m e x F$ was impaired (Fig. 4A). Furthermore, $\Delta m e x B$ and $\Delta m e x B \Delta m e x F$ mutants showed reduced production of AHL (Fig. 4B), suggesting that an RNDtype multidrug efflux pump transporter not only directly extrudes antimicrobials but also indirectly controls other types of virulence, such as surface motility and the quorum sensing system. This may be the result of environmental adaptation of the mutant strains in the absence of MexABOprM or MexEF-OprN.

\section{Acknowledgments}

We would like to thank the Leaf Tobacco Research Laboratory of Japan Tobacco Inc. for providing Pta 6605. This work was supported in part by JSPS KAKENHI Grant Numbers 16K14861 and 19H02956.

\section{Electric Supplementary Material}

Supplementary materials are available at The Plant Pathology Journal website (http://www.ppjonline.org/).

\section{References}

Alcalde-Rico, M., Hernando-Amado, S., Blanco, P. and Martínez, J. L. 2016. Multidrug efflux pumps at the crossroad between antibiotic resistance and bacterial virulence. Front. Microbiol. 7:1483.

Alvarez-Ortega, C., Olivares, J. and Martínez, J. L. 2013. RND multidrug efflux pumps: what are they good for? Front. Microbiol. 4:7.

Baker, C. J., Mock, N. M., Smith, J. M. and Aver'yanov, A. A. 2015. The dynamics of apoplast phenolics in tobacco leaves following inoculation with bacteria. Front. Plant Sci. 6:649.

Egea, C., Alcázar, M. D. and Candela, M. E. 1996. Capsidiol: its role in the resistance of Capsicum annuum to Phytophthora capsici. Physiol. Plant. 98:737-742.

Fan, J., Crooks, C., Creissen, G., Hill, L., Fairhurst, S., Doerner, P. and Lamb, C. 2011. Pseudomonas sax genes overcome aliphatic isothiocyanate-mediated non-host resistance in Arabidopsis. Science 331:1185-1188.

Fernando, D. M. and Kumar, A. 2013. Resistance-nodulationdivision multidrug efflux pumps in Gram-negative bacteria: 
role in virulence. Antibiotics 2:163-181.

Glazebrook, J. and Ausubel, F. M. 1994. Isolation of phytoalexindeficient mutants of Arabidopsis thaliana and characterization of their interactions with bacterial pathogens. Proc. Natl. Acad. Sci. U. S. A. 91:8955-8959.

Gnanamanickam, S. S. and Mansfield, J. W. 1981. Selective toxicity of wyerone and other phytoalexins to Gram-positive bacteria. Phytochemistry 20:997-1000.

Hernando-Amado, S., Blanco, P., Alcalde-Rico, M., Corona, F., Reales-Calderón, J. A., Sánchez, M. B. and Martínez, J. L. 2016. Multidrug efflux pumps as main players in intrinsic and acquired resistance to antimicrobials. Drug Resist. Updat. 28:13-27.

Ichinose, Y., Tasaka, Y., Yamamoto, S., Inoue, Y., Takata, M., Nakatsu, Y., Taguchi, F., Yamamoto, M., Toyoda, K., Noutoshi, Y. and Matsui, H. 2020. PsyR, a transcriptional regulator in quorum sensing system, binds lux box-like sequence in $p s y I$ promoter without AHL quorum sensing molecule and activates psyI transcription with AHL in Pseudomonas syringae pv. tabaci 6605. J. Gen. Plant Pathol. 86:124-133.

Ishiga, Y., Ishiga, T., Uppalapati, S. R. and Mysore, K. S. 2011. Arabidopsis seedling flood-inoculation technique: a rapid and reliable assay for studying plant-bacterial interactions. Plant Methods 7:32.

Kang, H. and Gross, D. C. 2005. Characterization of a resistancenodulation-cell division transporter system associated with the syr-syp genomic island of Pseudomonas syringae pv. syringae. Appl. Environ. Microbiol. 71:5056-5065.

Li, X.-Z., Plésiat, P. and Nikaido, H. 2015. The challenge of efflux-mediated antibiotic resistance in Gram-negative bacteria. Clin. Microbiol. Rev. 28:337-418.

Marutani, M., Taguchi, F., Shimizu, R., Inagaki, Y., Toyoda, K., Shiraishi, T. and Ichinose, Y. 2005. Flagellin from Pseudomonas syringae pv. tabaci induced hrp-independent HR in tomato. J. Gen. Plant Pathol. 71:289-295.

McClean, K. H., Winson, M. K., Fish, L., Taylor, A., Chhabra, S. R., Camara, M., Daykin, M., Lamb, J. H., Swift, S., Bycroft, B. W., Stewart, G. S. A. B. and Williams, P. 1997. Quorum sensing and Chromobacterium violaceum: exploitation of violacein production and inhibition for the detection of $N$ acylhomoserine lactones. Microbiology 143:3703-3711.

Morrissey, J. P. and Osbourn, A. E. 1999. Fungal resistance to plant antibiotics as a mechanism of pathogenesis. Microbiol. Mol. Biol. Rev. 63:708-724.

Sawada, T., Eguchi, M., Asaki, S., Kashiwagi, R., Shimomura, K., Taguchi, F., Matsui, H., Yamamoto, M., Noutoshi, Y., Toyoda, K. and Ichinose, Y. 2018. MexEF-OprN multidrug efflux pump transporter negatively controls $N$-acyl-homoserine lactone accumulation in Pseudomonas syringae pv. tabaci 6605.
Mol. Genet. Genomics 293:907-917.

Schäfer, A., Tauch, A., Jäeger, W., Kalinowski, J., Thierbach, G. and Pühler, A. 1994. Small mobilizable multi-purpose cloning vectors derived from the Escherichia coli plasmids pK18 and pK19: selection of defined deletions in the chromosome of Corynebacterium glutamicum. Gene 145:69-73.

Stoitsova, S. O., Braun, Y., Ullrich, M. S. and Weingart, H. 2008. Characterization of the RND-type multidrug efflux pump MexAB-OprM of the plant pathogen Pseudomonas syringae. Appl. Environ. Microbiol. 74:3387-3393.

Taguchi, F. and Ichinose, Y. 2011. Role of type IV pili in virulence of Pseudomonas syringae pv. tabaci 6605: correlation of motility, multidrug resistance, and HR-inducing activity on a nonhost plant. Mol. Plant-Microbe Interact. 24:1001-1011.

Taguchi, F. and Ichinose, Y. 2013. Virulence factor regulator (Vfr) controls virulence-associated phenotypes in Pseudomonas syringae pv. tabaci 6605 by a quorum sensing-independent mechanism. Mol. Plant Pathol. 14:279-292.

Taguchi, F., Inoue, Y., Suzuki, T., Inagaki, Y., Yamamoto, M., Toyoda, K., Noutoshi, Y., Shiraishi, T. and Ichinose, Y. 2015. Characterization of quorum sensing-controlled transcriptional regulator MarR and Rieske (2Fe-2S) cluster-containing protein (Orf5), which are involved in resistance to environmental stresses in Pseudomonas syringae pv. tabaci 6605. Mol. Plant Pathol. 16:376-387.

Taguchi, F., Ogawa, Y., Takeuchi, K., Suzuki, T., Toyoda, K., Shiraishi, T. and Ichinose, Y. 2006. A homologue of the 3-oxoacyl-(acyl carrier protein) synthase III gene located in the glycosylation island of Pseudomonas syringae pv. tabaci regulates virulence factors via $N$-acyl homoserine lactone and fatty acid synthesis. J. Bacteriol. 188:8376-8384.

Tsuji, J., Jackson, E. P., Gage, D. A., Hammerschmidt, R. and Somerville, S. C. 1992. Phytoalexin accumulation in Arabidopsis thaliana during the hypersensitive reaction to Pseudomonas syringae pv syringae. Plant Physiol. 98:1304-1309.

VanEtten, H., Temporini, E. and Wasmann, C. 2001. Phytoalexin (and phytoanticipin) tolerance as a virulence trait: why is it not required by all pathogens? Physiol. Mol. Plant Pathol. 59:83-93.

Vargas, P., Farias, G. A., Nogales, J., Prada, H., Carvajal, V., Barón, M., Rivilla, R., Martín, M., Olmedilla, A. and Gallegos, M.-T. 2013. Plant flavonoids target Pseudomonas syringae pv. tomato DC3000 flagella and type III secretion system. Environ. Microbiol. Rep. 5:841-850.

Vargas, P., Felipe, A., Michán, C. and Gallegos, M.-T. 2011. Induction of Pseudomonas syringae pv. tomato DC3000 MexAB-OprM multidrug efflux pump by flavonoids is mediated by the repressor PmeR. Mol. Plant-Microbe Interact. 24:1207-1219. 